\title{
AFFAIRE UNILEVER.DROIT DE TRAVAILLEURS DANS LES SOCIETES ETRANGERES
}

\author{
Par Stéphanie NSOMWE
}

\section{SUMMARY}

The company may know a transformation, however, will require that all transformations do not follow the traditional legal rules of the merger or acquisition as the purchase of all the shares of a company by another company.

Note that in our study we are interested in buying all the shares the company Marsavco that belonged to Unilever.

It is important above all to know that the Unilever Group was created in Congo(to day Democratic Republic of Congo: DRC) oil mills of the Belgian Congo, which later became Unilever Plantation in Zaïre (PLZ) with Zaïrianisation and then Unilever Plantation in Congo (PLC) with the advent of the AFDL in 1997.

This provided for palm oil to Marsavco for the manufacture of its products. And following the various rebellions or to the political crisis in the places where it had settled its plantations is to the north of the country, the PLC was cut off from its plantations and could not produce palm oil.

Hence the impossibility for Marsavco runs. She was therefore obliged to turn into selling all its shares in Rawji group that bought them all. The new Marsavco was born and then kept the same name. To comply with the requirements of the OHADA, the SA becomes Marsavco instead of SARL.

Having regard to all the above questions is at the heart of our concern is to know the fate of the former Marsavco workers in the new Marsavco.

For the answer to the question we made the descent to the place that is to say, the new Marsavco where we met their legal advisor who will inform us that Marsavco had to proceed with a selective criterion to terminate at amicable contract those deemed unproductive and those close in age to be retired. And in this they included termination clause that the transaction is conflict.

Though resolution mode, the Congolese Labour Code informs us in its Article 62 that can not terminate a contract for cause related to the ability, the need for operational or economic reasons (Art. 78). And it is clear from Article 63 of the Labor Code that dismissal which is not founded on any grounds mentioned in Articles 62 and 78 gives rise to reinstatement and failing to damages. Furthermore, Article 80 of the Labor Code informs us that when there is substitution of the employer all contracts in progress at the time of substitu-

1 Assistante à la faculté de droit de l'Université de Kinshasa, assistante du directeur technique dans une société des télécommunications,snsomwe@africell.cd ou stephaniensomwe@yahoo.fr. 
tion remain with the new employer. So we dare to think that the behavior of the new Marsavco frieze maliciously.Besides, redundant workers are complaining because their dismissal payment was not calculated as it should. Yet the new Marsavco had the opportunity to build on the mentioned economic reasons or by Art. 62 either by s. 78 of the Labour Code to dismiss en masse those who should leave.

\section{INTRODUCTION}

Les préoccupations d'ordre économique et politique avaient poussé les puissances européennes et américaines à se réunir en vue de discuter sur la question de leurs intérêts en Afrique. C'est alors que « le 15 novembre 1884 s'ouvrait à Berlin la Conférence internationale dont les travaux devaient aboutir à la signature de l'Acte de Berlin, du 26 février 1885. ${ }^{2} \gg$

« Dans son discours d'inauguration, le prince de Bismarck s'exprimait ainsi : l'idée fondamentale du programme de la Conférence est de faciliter à toutes les nations commerçantes l'intérieur de l'Afrique. $»^{3}$

« Depuis quelques années, un changement s'opérait dans l'équilibre économique des Etats européens. L'ancienne doctrine de l'école de Manchester, le libre- échange, reconnue bonne et appliquée en partie en Europe depuis 1861, était abandonnée successivement par la France, l'Allemagne, l'Italie. Les doctrines protectionnistes succédaient aux doctrines libre-échangistes et devenaient dominantes en ces pays. $»^{4}$

« Or, il est permis de l'affirmer, il n'y a pas seulement, entre l'inauguration du système protectionniste en Europe, et l'annexion européenne de l'Afrique, simple coïncidence de date, mais aussi relation étroite de cause à effet. $»^{5}$ " La France, l'Allemagne, qui, les premières, avaient, adopté ce système, étaient naturellement amenées, pour compenser la restriction des échanges résultant du protectionnisme, à chercher des marchés spéciaux. ${ }^{6}$

« La Grande-Bretagne, qui veut avant tout avoir le monde libre devant elle pour faire des échanges, se voyait tout à coup menacée par cette attitude dans ses intérêts les plus chers. Car le commerce est, pour l'Angleterre, une question de vie ou de mort. La politique anglaise n'est pas déterminée par des préférences ou des passions de circonstances. Elle est la résultante d'un ensemble de nécessités nationales. La terre anglaise, répartie entre un petit nombre de possesseurs, ne suffit pas à la nourriture de la population; la production industrielle anglaise est surabondante pour la consommation d'un peuple confiné en ses îles; ces îles elles- mêmes sont trop petites pour la multiplication d'une race féconde. Pour pouvoir vivre, les Anglais ont donc dû se répandre partout, constituer leur île en grande métropole,

2 Le $Q R 9 / N P(J$.$) , La liberté de commerce dans le bassin conventionnel du Congo, Paris, p.3.$

3 Le QR9/NP (J.), note1, p.3.

4 Le $Q R 9 / N P(J$.$) , note1, p.3.$

5 Le $Q R 9 / N P(J$.$) , note1, p.3.$

6 Le $Q R 9 / N P(J$.$) , note1, p.4.$ 
et s'ouvrir dans le reste du monde de vastes débouchés où ils déversent l'excédent de leur population, d'où ils tirent les aliments qui leur manquent, où ils envoient leur surproduction industrielle. L'idéal politique anglais est donc l'ouverture des marchés.» ${ }^{7}$

« La Belgique enfin, qui voyait se fermer devant elle les marchés continentaux, cherchait ailleurs des débouchés. Dès 1860, le major de Brialmont réclamait comme une nécessité pour son pays l'établissement de colonies, afin de remplacer le vaste marché que lui offriraient avant 1830 les possessions néerlandaises. Depuis, la situation avait empiré. Tant que le libre-échange a été pratiqué par l'Europe et l'Amérique, la situation de la Belgique fut très pros- père. Grâce à leur outillage économique, ses manufactures purent lutter avec avantage sur tous les marchés. Le mouvement du port d'Anvers, pouls de l'activité économique du pays, s'était notablement accru : c'était le premier port du continent. Mais les grandes nations renoncèrent au libre-échange pour y substituer le régime de la protection. Or celle-ci est fatale aux petits pays qui ne peuvent user de représailles envers les grands, leur faire une guerre de tarifs, sous peine d'être écrasés. Aussi la situation de l'industrie belge devenait-elle très précaire : le commerce général du pays diminuait; le mouvement du port d'Anvers était peu à peu dépassé par celui de Hambourg, et la population s'accroissant sans cesse, une crise sociale s'imposait si l'on n'ouvrait pas des contrées nouvelles à l'activité des travailleurs $»^{8}$.

Aussi, il faudra noter que « ce n'est pas seulement à des considérations économiques qu'obéissaient les Puissances réunies à Berlin. Elles avaient des visées politiques plus hautes. A cette même époque où les nations s'orientaient dans un sens économique tout nouveau, les explorations de Stanley et de Brazza vinrent brusquement révéler l'existence, au centre de l'Afrique, de régions susceptibles d'une exploitation rémunératrice. Le roi des Belges, Léopold II, prévoyant le parti que l'on pourrait en tirer, convoque en 1876, à Bruxelles, les présidents de sociétés de géographie de divers pays, pour délibérer sur « les conditions auxquelles la civilisation pourrait pénétrer en Afrique». On crée une «Association internationale africaine ", société à caractère privé, ayant des comités nationaux, et dont le but est de créer des stations internationales pour secourir les explorateurs, approvisionner les missions, protéger les indigènes. Les comités nationaux se montrèrent sans grande activité pour d'autres œuvres que les leurs. Peu à peu ils se détachèrent de l'Association qui dès lors évolue dans un sens économique, commercial, territorial. ${ }^{9}$

Il conviendrait de savoir que cette association internationale n'était créée que pour ménager l'opposition belge et les susceptibilités étrangères. Le roi a voulu tout simplement se servir de ce cachet international pour échapper aux jalousies des grands Etats européens, aux compétitions et aux obstacles. Et au congrès de Berlin l'œuvre royale devient un Etat sous la protection de l'Europe.

7 Le QR9/NP (J.), note1p.5.

8 Le QR9/NP (J.), note1, p.5.

9 Le $Q R 9 / N P(J$.$) , note1, p.8.$ 
«En 1882, il devient 1' « Association internationale du Congo ». Son activité est prodigieuse; Stanley ne se contente pas de passer plus de quatre cents traités avec les indigènes : il explore le bassin du Congo, il crée Vivi, fonde Léopoldville, trace des routes, s'assure un débouché sur la côte par la vallée du Kouilou-Niari. »Effrayé de ses progrès incessants, les Puissances (La France et L'Allemagne) et les sociétés commerciales de tous les Etats commencèrent à se rendre compte de riches contrées du Congo. ${ }^{10}$ " Des compétitions étaient donc à craindre, malgré tout. Chaque pays intéressé allait vouloir, en accroissant ses territoires, se réserver le monopole de richesses qu'on devait sans doute découvrir. C'est afin d'éviter ces complications qu'on songea à instituer pour les contrées comprises sous le nom de «Bassin Conventionnel du Congo », une sorte de neutralité, ... » Et le 2 février 1885 fut signé par tous les Etats maritimes d'Europe et les États-Unis d'Amérique, l'Acte général de la Conférence de Berlin qui statuait sur le régime économique d'un vaste territoire comprenant non seulement le bassin géographique du Congo, mais encore ses tenants et aboutissants naturels, de l'Océan Atlantique à l'Océan Indien, soit une superficie de 6.250.000 kilomètres carrés, douze fois celle de la France.

L'Association internationale en détenait la majeure partie; la France, le Portugal, l'Egypte, l'Angleterre, l'Allemagne, le sultanat de Zanzibar y avaient aussi des possessions. En ce qui concerne le Gabon, le bassin de l'Ogooué est exclu du bassin conventionnel, mais celui du Kouilou-Niari y est rattaché.

L'article 1 de l'acte de Berlin dispose Le commerce de toutes les nations jouira d'une complète liberté au Congo.

Ce principe ne rien d'autre que le principe de la liberté de commerce et industrie. Il n'était pas nouveau, il était issu du décret d'Allarde de 2 et 17 mars 1791selon lesquelles toute personne est libre d'exercer telle profession, art, ou métier qu'elle trouvera bon.

Pour mettre en valeur ses vastes territoires, l'Etat Indépendant a employé toutes les méthodes; mise en exploitation directe par l'État, puis exploitation par des compagnies à privilèges, et, en dernier lieu, système des grandes et moyennes concessions foncières accordées à des sociétés puissantes, se chargeant; soit de l'exploitation proprement dite, soit de l'exécution de grands travaux d'utilité générale. ${ }^{11}$

C'est ainsi que l'on octroya à l'anglais William Lever en 1911 des vastes concessions dans la partie centrale du pays pour la production de l'huile de palme. «Bien qu'il a été dit lorsque la Belgique reprend le Congo, qu'il n'y aurait plus de grandes concessions territoriales, le gouvernement belge accorde, moyennant certaines conditions, à une société étrangère (juridiquement belge mais à capitaux majoritairement anglais), des droits pouvant conduire à terme à l'obtention en propriété de 750000 ha de terres, soit l'équivalent d'un quart de la superficie de la Belgique et un monopole de fait pour l'exploitation de palmeraies sur plus de $39000 \mathrm{~km}^{2}$, soit l'équivalent de 1,3 fois cette superficie. $»^{12}$

10 Le $Q R 9 / N P(J$.$) , note1, p.10.$

11 Le QR9/NP (J), note 1, p.10.

12 Belgeo, https://belgeo.revues.org/11572, consulté le 1 mars 2016. 
Cependant, La production de l'huile de palme démarre vraiment avec l'installation de la firme Lever Brothers Limited, une des plus grosses entreprises anglaises de savon et de margarine qui fusionnera en 1929 avec l'anglo-hollandaise Margarine Union-Margarine Unie pour constituer la multinationale Unilever.

Ce groupe Unilever avait à Kinshasa depuis 1922, outre la huilerie du Congo Belge $(\mathrm{HCB})$ une autre entreprise qui fabriquait le savon et la margarine dénommée Marsavco (margarine et savon du Congo).

Malgré son succès, cette société finit par être confrontée à des divers problèmes qui l'ont poussé à vendre ses actions au profit du groupe Rawji. Nous y reviendrons plus tard.

La question la plus préoccupante à ce sujet est celle de savoir quel sera le sort des salariés de la Marsavco sous le groupe Unilever dans le groupe Rawji.

Pour répondre à cette préoccupation nous avons jugé de subdiviser notre étude en deux points. Il s'agira dans le point A de la multinationale et dans le point B de la transformation d'une société et le droit des travailleurs congolais dans la nouvelle marsavco.

\section{A. La multinationale et le groupe de sociétés en droit ohada}

Généralement les entreprises de petites tailles se sentent en insécurité lorsque les entreprises de grandes tailles connaissent un essor vertigineux.

« Les petites entreprises, au coût de production élevé, se voient menacées par cette évolution. Leur seule issue consiste à se grouper, à fusionner et à conclure une entente, éventuellement avec des grandes entreprises de la même industrie. $»^{13}$

\section{Notions de la multinationale}

1. Définition d'une multinationale et d'une filiale

On définira comme entreprise multinationale « toute entreprise originaire d'un pays ayant des activités stables et sous son contrôle dans au moins deux pays étrangers, et où elle réalise plus de $10 \%$ de son chiffre d'affaires. $\rangle^{14}$

Cette entreprise d'origine s'appelle société mère et la société implantée dans le pays étranger s'appelle filiale.

«L'entreprise du pays d'origine s'appelle la maison mère, et ses extensions dans le pays d'accueil des filiales étrangères. $»^{15}$

Une société mère n'est pas toujours l'œuvre d'un seul propriétaire. Plusieurs sociétés peuvent se grouper pour constituer une filiale afin de contrôler un marché.

13 Serges (J.), Les conditions de la croissance économique, Kinshasa-Zaïre, Sept.1973, p.66.

14 Ghertman(M.), Les multinationales, févr.1985, p.4.

15 Ghertman(M.),note1,p.4. 
Le régime des nombreuses entreprises, soumises à la loi de la concurrence, cède la place à un capitalisme de groupes, qui aspirent à dominer le marché, et qui sont dirigés par quelques « chef d'industrie » ${ }^{16}$

« Il arrive fréquemment que la filiale n'est pas l'œuvre d'une seule société-mère et que deux ou trois sociétés se groupent pour constituer dans leur intérêt privé une filiale unique. $»^{17}$ «Par contre une même société peut créer plusieurs filiales. ${ }^{18}$

\section{Objectifs}

Une multinationale a pour objectif la survie, le profit et la croissance

\section{a. La survie}

L'entreprise est un organisme vivant dont le seul but n'est pas de faire gagner de l'argent à l'entrepreneur et à ses actionnaires. Elle doit également rémunérer les banquiers ${ }^{19}$ qui lui prêtent de l'argent et l'Etat auquel elle paie des impôts; elle doit aussi faire vivre des fournisseurs, des employés, des ouvriers, et alimenter en produits et en services des clients qui en ont besoin pour leur propre survie. ${ }^{20}$

\section{b. Le profit}

Toute organisation a besoin d'être rentable,sinon,elle a plus de difficultés que ses concurrents pour trouver des actionnaires et des banques qui prêtent de l'argent pour investir en recherches et en usines modernes, payer les fournisseurs et les employés suffisamment pour obtenir une contribution d'un haut niveau. ${ }^{21}$

\section{La croissance}

Si une entreprise ne croît pas au même rythme que ses concurrents nationaux et étrangers, ceux-ci, vont lui prendre sa part de marché. Avec un affaiblissement de celle-ci, il n'y aurait plus de profit, plus de survie. ${ }^{22}$

$16 \operatorname{Ghertman}(M$.$) , note1, p.4.$

17 Moreau (A.), La société anonyme, Traité Pratique, Paris, p.1083.

18 Moreau (A.), note1, p.1083.

19 Ghertman (M.), note1, p.36.

20 Ghertman (M.), note1, p.37.

21 Ghertman (M.), note1, p.19.

22 Ghertman (M.), note1, p.20. 


\section{Le groupe de sociétés en droit ohada}

1. «La reconnaissance de groupe de sociétés en droit ohada.

Les entreprises africaines, petites, moyennes et grandes peuvent ainsi concentrer leurs moyens afin d'accroître leur part de marché, tout en conservant chacune son indépendance juridique.

L'appartenance à un groupe peut présenter de nombreux avantages pour une société. Elle est de nature à lui procurer des appuis financiers, des approvisionnements plus faciles, plus réguliers et moins coûteux, des débouchés plus importants, etc. L'unité de direction du groupe assure aussi une coordination de l'activité des différentes sociétés, propice au développement de la productivité et la spécialisation. Tous ces avantages expliquent la puissance économique et financière, souvent décriée, mais toujours reconnue des grandes firmes multinationales dont les filiales sont implantées à travers le monde. La reconnaissance du groupe de sociétés par législateur africain témoigne également de cette importance. Ainsi, aux termes de l'article 173 de l'Acte uniforme sur les sociétés commerciales et du GIE, le groupe désigne « l'ensemble formé par des sociétés unies entre elles par les liens divers qui permettent à l'une d'elles de contrôler les autres ». La notion de contrôle entendue largement comme « la détention effective du pouvoir de décision au sein d'une société », peut résulter de la détention directe ou indirecte de plus de la moitié des droits de vote d'une société, notamment en vertu d'accords conclus avec d'autres associés de la société contrôlée. Le droit OHADA a permis de déterminer le cadre juridique du développement des groupes des sociétés, à travers la réglementation des participations, des comptes consolidés et des comptes combinés. Tout en ménageant un cadre propice aux investissements directs étrangers, le droit OHADA vise aussi à doter les économies des pays membres de structures d'intégration économique. A cet effet, l'Acte uniforme sur les sociétés commerciales autorise la création du groupe réunissant des sociétés juridiquement indépendantes et implantées dans différents pays membres de l'OHADA.L'article 84 permet à une société dont le siège est situé dans l'un des Etats parties de faire un appel public à l'épargne dans tous les autres Etats membres.

Dans un contexte de mondialisation de l'économie on peut aisément concevoir la puissance commerciale d'un groupe de sociétés résultant de la concentration des entreprises africaines productrices de telle ou telle matière première. On peut même envisager des cas de concentration verticale où le groupe ainsi constitué aurait la maîtrise totale d'un secteur d'activité, en assurant la fabrication d'un produit d'extraction des matières jusqu'à la vente aux consommateurs. Ce schéma peut parfaitement être utilisé pour des produits tels que le café, le cacao, coton, bois, l'arachide, l'hévéa... et les ressources minières. C'est le sens qu'il faudrait donner à l'intégration économique en Afrique. C'est surtout de cette façon que l'Afrique deviendrait actrice et non plus simplement victime de la mondialisation. Toutefois, dans son fonctionnement le groupe de sociétés peut présenter quelques risques pour les associés minoritaires des filiales : les bénéfices réalisés par certaines filiales sont intégrale- 
ment remontés vers la société mère par le jeu de divers contrats l'émergence des égoïsmes nationaux pourrait constituer un véritable obstacle à l'intégration économique régionale.

Une meilleure protection des associés minoritaires des filiales passe par la soumission des conventions intra groupe au même régime que celles conclues entre une société et ses dirigeants. $\gg 23$

2. Le régime juridique d'un groupe de société et d'une société en droit ohada.

Il convient de savoir que les groupes de sociétés n'ont pas de personnalité juridique par contre ce sont leurs filiales qui sont dotées d'une personnalité juridique. Ainsi, il importe de l'étudier par rapport à leurs filiales établies sur l'espace ohada.

a. La personnalité morale d'une société sur ohada

«A l'exception de la société en participation, toute société doit être immatriculée au Registre du commerce et du crédit mobilier(RCCM).» ${ }^{24} \mathrm{Et}$ l'article 98 de l'Acte Uniforme dispose « toute société jouit de la personnalité juridique à compter de son immatriculation au registre du commerce et du crédit mobilier, à moins que le présent Acte Uniforme ne dispose autrement. $»^{25}$

b. Le statut de la société sur ohada

L'article 13 de l'Acte Uniforme précise que : «Les statuts énoncent :

$1^{\circ}$ La forme de la société;

$2^{\circ}$ Sa dénomination suivie; le cas échéant, de son sigle;

$3^{\circ}$ La nature et le domaine de son activité, qui forment son objet social ....etc. ${ }^{26}$

c. La nationalité

« Toute société doit être localisée dans un lieu appelé siège social, qui doit être mentionné dans les statuts. Cette localisation emporte des conséquences notamment en ce qui concerne

23 Lelo-Di-Makangu (U.), La capitalisation du Groupement d'Intérêt Economique de 1'Ohada, http:// www.mémoireonline.com, consulté le 04 mars 2016.

24 Article 97 de l'Acte Uniforme relatif au droit des sociétés commerciales et du groupement d'intérêt économique.

25 Article 98 de l'Acte Uniforme relatif au droit des sociétés commerciales et du groupement d'intérêt économique.

26 Alioune Dièye, Régime juridique des sociétés commerciales et du GIE dans 1'espace ohada, 2014, p.69. 
la compétence territoriale. En principe, c'est le siège social qui détermine la nationalité d'une société. $»^{27}$

«Une société est régie par la loi nationale du moins pour les règles constitutives et de fonctionnement de la société. $»^{28}$

Il y a des difficultés à déterminer la nationalité de la société. « Le critère de siège social réel est d'abord dégagé par la jurisprudence. Cette solution est confirmée par l'article $1^{\mathrm{er}} \mathrm{de}$ l'Acte Uniforme qui, il est, n'emploie pas le terme «nationalité » ${ }^{29}$. "Ainsi, une société dont le siège social est au Sénégal est soumise aux lois non contraires à l'Etat partie où se situe le siège social.» ${ }^{30}$

Notons que les groupes de sociétés et les sociétés dont il s'agit ici concernent, ceux africains et dont les pays se sont inscrits sur l'espace ohada. Toute fois une société ou une filiale même appartenant aux sujets étrangers est considérée comme de la société nationale et est soumise aux règles de l'ohada dont fait partie l'Etat où se situe son siège social.

Ainsi, la société marsavco bien qu'appartenant au sujet indien est soumise pour d'autres aspects aux règles dont fait partie l'Etat où elle a établi son siège social. La RDC est membre de l'ohada.

Il est très utile de savoir qu'une société peut être soumise à certaines opérations juridiques sous-entendues sous le mot de «transformation par concentration » tels la fusion, la scission, la prise de participation et les groupes des sociétés.

Etant donné que la marsavco n'est pas une filiale appartenant à un groupe africain de l'espace ohada, nous ne l'étudierons pas par rapport à la transformation des sociétés en droit ohada mais par rapport au droit commun régissant les sociétés en RDC.

\section{B. La transformation d'une société et le droit des travailleurs dans la société marsavco}

Lorsqu'une société ne génère plus des bénéfices comme il se doit ou lorsqu'elle est en carence de matières premières lui permettant de réaliser les opérations pour lesquelles elle a été créée (objet social), il peut arriver qu'elle subisse des mutations profondes. Ces mutations peuvent donner lieu à plusieurs opérations juridiques.

Ainsi, bien que lacunaire parce que ne prévoyant pas la possibilité d'une société unipersonnelle, point n'est la contradiction sur le fait que l'article 446 al.1 du Code civil livre III, en nous définissant la société comme « un contrat par lequel deux ou plusieurs personnes conviennent de mettre quelque chose en commun en vue de partager le bénéfice qui pourra en résulter» ${ }^{31}$, nous révèle que la société est créée pour réaliser le bénéfice sans lequel elle

27 Alioune Dièye, Régime juridique des sociétés commerciales et du GIE dans l'espace ohada, 2014, p.50.

28 Alioune Dièye, note1, p.50.

29 Alioune Dièye, note1, p.50.

30 Alioune Dièye, note1, p.50.

31 Art. 446 du Code civil congolais LIII. 
est appelée à disparaître. Pour John Van Damme le contrat de société est « une convention par la quelle deux ou plusieurs personnes conviennent de former un fond commun, au moyen de mise à fournir par chacune d'elles, dans la vue de partager les bénéfices ou les pertes qui pourrons en résulter $»^{32}$

En effet une société peut subir des transformations suite à la fusion ou absorption, scission et autres mais il existe des transmutations qui n'obéissent à ces règles telles la cession d'une fraction de l'avoir social, l'obtention par les associés de la société disparue à titre de contrepartie, des espèces et obligations en lieu et place des parts sociales ou actions et l'achat par une société de toutes les parts sociales.

\section{La transformation d'une société commerciale en droit de société congolaise}

Etant donné que la marsavco n'est pas une société appartenant au groupe des sociétés africaines membres de l'ohada, ses règles ne méritent pas de lui être appliquées. Pourtant, en se basant sur la nationalité, la marsavco est considérée comme une société congolaise sur base du critère de siège. Cela étant elle doit en principe être soumise à certaines réglementations de l'ohada car la RDC en est membre.

Si les règles relatives à la transformation de la société en droit ohada ne peuvent être applicables à la marsavco du fait qu'elle n'est pas une société du groupe africain de l'espace ohada, notons que certaines de ses règles lui seront appliquées en raison de son appartenance à la RDC.

« Notons que la transformation est une opération qui a but de convertir une société soumise à un régime légal déterminé en une société d'un autre régime soit par changement de type, soit par changement de nationalité. ${ }^{33}$ Et pour M. Wahl, « en principe, la transformation d'une société en une société d'un autre type emporte dissolution de la société qui se transforme et la création d'une société nouvelle. La loi, en effet, établit divers types de sociétés qui se distinguent les unes des autres et qui ont chacune une personnalité morale. $»^{34}$

Pourtant, outre la fusion ou absorption il existe d'autres transformations qui ne répondent aux règles habituelles de la transformation.

1. «Les Transmutations qui ne répondent pas au concept juridique de fusion ou d'absorption.

Certaines transformations ne répondent pas au concept juridique de fusion ou d'absorption. On peut citer des exemples à titre d'illustration.

32 John Van Damme, Extraits des études sur les sociétés commerciales, in revue de doctrine et de jurisprudences coloniales, 1926, pp.693-813.

33 Moreau (A.), note 1, p.1105.

34 Wahl(M.), De la transformation des sociétés, 1910, p.97. 


\section{a. Cession d'une fraction de l'avoir social}

Lorsqu'une société apporte seulement une partie de son actif (un département de ses activités multiples par exemple) à une autre société, on parle d'apport partiel d'actif.

La fusion(ou d'absorption) exige qu'un ensemble de biens et de charges c'est-à-dire une universalité soit reprise. La cession d'une fraction de l'avoir social, si massive fut-elle, voire la cession d'une branche complète d'activité, reste une vente. La société qui s'ampute de la sorte survit alors que la fusion ou l'absorption ne laissent aucune vie propre à la société fusionnante ou absorbée. Si la personnalité se prolonge, c'est entièrement au sein de la société absorbante.

b. L'obtention par les associés de la société disparue, à titre de contrepartie, des espèces et obligations en lieu et place des parts sociales ou actions.

Il n’y pas de véritable absorption si les associées de la société disparue reçoivent non des actions ou parts sociales mais des espèces ou des obligations.

c. Achat par une société, de toutes parts sociales ou actions d'une autre société

L'achat par une société de toutes les actions ou parts sociales d'une autre société, entraînant la disparition de la seconde société, n'est pas une véritable fusion. Il manque, en effet, à la fusion, une de ces caractéristiques essentielles, l'attribution aux associés de la société absorbée d'actions ou parts de la société absorbante.. ${ }^{35}$

Par ailleurs il sied de noter que nous portons notre attention sur l'achat de toutes les parts sociales car il répond à notre préoccupation dans le cadre de cette étude

En outre, nous nous intéressons à l'achat de toutes les actions de la marsavco par le groupe Rawji.

Avant d'entrer dans le vif du sujet nous allons retracer un petit historique de la marsavco.

2. Historique de la marsavco et effet du changement sur la personnalité morale et le statut de la nouvelle marsavco

La Marsavco,en sigle(La société des margarines,savonneries et cosmétiques) SARL créée en 1922, le 16 février par le groupe britanique Lever Brothers,sous raison sociale SAV$\mathrm{CO}$ (savonnerie du Congo) en vue de la fabrication des savons à partir de l'huile de palme et palmistes de la société huilerie du congo(HCB).En 1922, le groupe Lever Brothers,de Londres et Margarine Unie de Rotterdam fusionnent pour créer le groupe « Unilever » qui fut la maison mère de l'actuelle Marsavco.

35 Lukombe Ghenda, Droit congolais des sociétés, Kinshasa, 1999, p.967. 
Suites aux changements politiques intervenants dans notre pays et à l'évolution de son objet social, la marsavco a dû connaître des transformations progressives de 1929-1952.

En 1963, elle a connu un essor considérable avec l'acquisition d'une chaine de fabrication des pâtes dentifrices et des poudres détergentes et à récurer.

En 1972, elle devient Marsavco-Zaïre avec la zaïrianisation et en 1976 elle fut rétrocédée au groupe Unilever avec la rétrocession. Et le 17 mai 1997, elle redevient marsavco.

Pendant la période allant de 1998 à 2002, la marsavco avait fait une chute aux enfers suite aux guerres de rébellion et des libérations qu'ont connues certaines provinces de notre pays. Elles l'avaient coupée de ces principales sources d'approvisionnement en huile de palme situées pour la plupart dans les territoires autrefois occupées par les forces rebelles.

Cette situation l'a conduite à vendre toutes ses parts au groupe Rawji.Et celui-ci a dû garder la même dénomination sociale et la même forme.

Pour s'adapter aux nouvelles exigences du droit ohada, elle a dû s'immatriculer au nouveau registre de commerce et de crédit mobilier (CD/KIN/RCCM/13-B-0893) afin d'acquérir de la personnalité juridique. Et elle a la forme de S.A. aujourd'hui conformément à l'ohada. «S'il n'y a pas création d'une société nouvelle, l'ancienne société ne se trouve pas dissoute et l'être moral continue à subsister...» ${ }^{36}$ " La pratique, plus libérale, estime que la transformation d'une société ...n'implique pas nécessairement la dissolution de la société qui, comme on le dit, change seulement « d'habit » la personne restant la même. » ${ }^{37}$

Sommes toute, nous allons retenir que la marsavco a gardé le même statut que celui de l'ancienne marsavco mais avec quelques adaptations par rapport aux exigences du droit ohada. Donc la marsavco continue à subsister.

Au-delà de toutes ces considérations la préoccupation reste unique. L'implication de ces changements sur le sort des travailleurs sous le groupe Unilever.

\section{B. La protection des travailleurs au regard de l'art.80 du code de travail}

La Déclaration universelle des droits de l'homme de 1948 consacre la liberté du travail, le droit au travail, à des conditions équitables et satisfaisantes, à la sécurité sociale, à la protection contre le chômage, à une rémunération équitable et conforme à la dignité humaine, la liberté syndicale. ${ }^{38}$ " Tout en adhérant à ces dispositions, un Etat est appelé à créer des emplois pour sa population en prenant toutes les mesures pouvant garantir à chaque travailleur les meilleures conditions de travail. $»^{39}$

36 Moreau (A.), note 1, p.1106.

37 Moreau (A.), note 1, p.1106.

$38 \operatorname{Verdier}(J M)$, Coeuret(A), Souriac $(M-A)$, Droit du travail, Rapports collectifs, Volume1, Paris, 2005, p. 25 .

39 Stéphanie Nsomwe, réflexion sur la protection de l'emploi dans le cadre de la sous-traitance au regard du code du travail en RDC. Cas de travailleurs assujettis par la sous-traitance dans le secteur de télécommunications, Librairies KAS, 2015, p.1. 
Les dispositions de la Déclaration universelle des droits de l'homme susmentionnées ont pour objectif de protéger les emplois. ${ }^{40}$ Et la protection de l'emploi et le respect de la dignité humaine sont garanties par la Constitution de la RDC. ${ }^{41}$

Notons que le secteur du travail mérite une attention particulière sur le plan législatif et exige une consécration particulière vue la pertinence de cette structure. ${ }^{42}$ " De tout temps, le travail est considéré comme la clé de tout développement socio-économique; toutes les sociétés recourent à ce moyen afin de faire face aux aléas de la vie et de garantir un avenir plus ou moins meilleur en se mettant à l'abri des risques. ${ }^{43}$ » Et aussi « Le travail qu'il soit manuel ou intellectuel est un besoin vital- un moyen pour l'homme de s'accomplir. $»^{44}$

Toute personne physique ou morale de droit privé qui utilise les services d'un ou de plusieurs travailleurs en vertu d'un contrat de travail est concernée par le droit du travail, et ses rapports avec les travailleurs utilisés sont soumis au code du travail. ${ }^{45}$ Le $~ «$ Le Code du Travail mérite d'être considéré comme un instrument capable d'apporter la paix sociale grâce à l'affermissement des relations professionnelles, au rétablissement des droits fondamentaux du travailleurs et de l'entrepreneur que sont le droit au travail et la liberté d'entreprise. ${ }^{46}$

Mais ce droit au travail ne serait que leurre s'il n'était pas assorti d'une protection résultant des mesures publiques contre le dénuement économique et social dans lequel la maladie, la maternité, les accidents de travail, le décès, etc. plongerait les travailleurs. En effet, le caractère social du travail, en ce qu'il procure un revenu au travailleur pour subsister, doit être stabilisé par les pouvoirs publics, afin d'éviter l'arbitraire et la loi du plus fort. ${ }^{47}$

Ainsi que le soulignent les professeurs Camerlynck et Lyon-Caen, le droit du travail dans tous les aspects, est sous la coupe de la conjoncture et de l'infrastructure économique. ${ }^{48}$

« Toutes les questions traitées par le droit du travail tendent à l'amélioration de la condition du travailleur. La plupart de ces questions gravitent autour des charges sociales chiffrables. Celles-ci comprennent, outre les salaires, tout ce que l'emploi coûte à l'entreprise (soins médicaux, congés, économats, hygiène et salubrité sur les lieux de travail,muta-

40 Stéphanie Nsomwe, note1, p.1.

41 Lire les art.36 et 16 de la constitution de 2006 de la RDC.

42 Lire les art.36 et 16 de la constitution de 2006 de la RDC.

43 Lire l'avant-propos de Kumbu ki Ngimbi, Droit du travail, manuel d'enseignement, Kinshasa, 2010, p. v.

44 Masanga Phoba Mvioki (J.), note1, p.2.

45 Masanga Phoba Mvioki (J.), note 1, p.4.

46 Code de travail, http : // www.collectif24.org/lib/documents/code_travail-2.pdf consulté le 10 octobre 2014.

47 Kumbu ki Ngimbi, note1, p.v.

48 Luwenyema Lule, «Précis de droit du travail congolais », Kinshasa, 1987, p.16. 
tion ect).Bref le droit du travail dans son ensemble grève le prix de revient de la maind'oeuvre. $)^{49}$

« De cette situation apparaît inévitablement une opposition d'intérêts entre l'employeur, qui lui, cherche à minimiser le coût de production, et les travailleurs qui réclament toujours de nouveaux avantages. $»^{50}$

Notons que « le droit du travail a pour souci majeur de consacrer tout ce qui est socialement souhaitable, mais par contre il ne peut réaliser que ce qui est économiquement possible. ${ }^{51}$ Concernant sa technique, nous constatons que le droit du travail a une finalité propre. Il est orienté vers la protection des salariés; donc une finalité qui inspire le contenu des règles relatives au travail-salaire, leur interprétation doctrinale et jurisprudentielle dans le sens le plus favorable aux salariés. ${ }^{52}$

Pour ce faire, il importe d'appliquer le texte du Code du travail quant en ce qui concerne le contrat de travail et le salaire. ${ }^{53}$

\section{Effet de la transmutation sur le droit des travailleurs avec la nouvelle marsavco}

Comme évoquée déjà ci-haut,la transmutation dont il est question ici concerne l'achat de toutes les parts sociales du groupe Unilever par le groupe Rawji.

En effet,en vertu du principe civiliste l'actif et le passif passent entre les mains du nouvel acquéreur, les droits des travailleurs doivent subir automatiquement une mutation avec cette transformation.

Mais qu'en est-il de la situation des travailleurs de l'ancienne marsavco?

La nouvelle marsavco a dû procéder par la selection des travailleurs qu'elle a estimé bon pour la séparation à l'amiable et ceux qui devaient continuer avec leur contrat au sein de l'entreprise.Le critère selectif tient compte de la perfomance du travailleur(le travailleur improductif doit être écarté.) et de l'âge d'être mis à retraite. Selon elle, les moyens dont elle dispose ne le justifie pas à reprendre tous les travailleurs.

Après avoir prouvé qu'elle a fait tout le nécessaire en empruntant même de l'argent ça et là,elle n'a pas su toujours comment les prendre en charges et sur ce, elle se voit dans l'obligation de dimuer l'effectif. Pour ce faire, elle a dû proposer une séparation à l'amiable qui est en fait un compromis avec le travailleur.Dans leur accord ils conviennent d'inclure dans la résiliation du contrat la clause transactionnelle qui leur permet de se séparer à l'amiable et de transiger sur les éventuels différends. Il importe de savoir que pour la marsavco,cette clause n'a acquis une force de droit qu'à partir du moment où l'inspecteur du travail l'a certifié sur un procès verbal.Et en ce moment même elle revêt le caractère de

Luwenyema Lule, note 1, p.15.

50 Luwenyema Lule, note 1, p.15.

51 Luwenyema Lule, note 1, p.15.

52 Masanga Phoba Mvioki (J.), note 1, p.24.

53 Stéphanie Nsomwe, note 1, p.1. 
l'autorité de la chose jugée à l'égard de deux parties au contrat. Cependant une tierse personne peut s'opposer à cette clause.Selon elle, pour que cette clause ne soit pas attaquable par le tiers, elle a dû prendre un jugement d'expédiant accordé par le tribunal du travail.

Le comportement du nouvel employeur peut paraître comme de la ruse car pour quelqu'un qui a eu à réaliser plusieurs années de service,le critère de perfomance ne vaut.Il y a manque d'objectivité dans cette sélection.En réalité l'on ne sait pas exactement sur base de quel critère il fonde son argument pour sélectionner. ${ }^{54}$ Aussi quand bien même qu'il aurait payé le décompte final,les anciens travailleurs se plaignent sur le fait qu'il n'était pas calculé comme il se doit. Quant aux travailleurs proche de l'âge d'être mis en retraite,l'on ne doit pas les anticiper sous pretexte que l'entreprise n'a pas assez de moyens.Nous osons croire qu'on devrait le laisser continuer leurs contrats tranquillement jusqu'à arriver à leur terme.

Il importe de savoir que les droits des travailleurs de l'ancienne marsavco sont ignorés par la nouvelle marsavco dirigée par le groupe Rawji quand bien même qu'ont leur avaient payer leur décompte final. D'où il importe de chercher à savoir ce que le code du travail prévoit.

\section{La protection des travailleurs au regard du code de travail congolais}

L'art. 80 du code de travail dispose: «Lorsqu'il y a substitution d'employeur, notamment par cession, succession, fusion, transformation de fonds, mise en société, tous les contrats de travail en cours au jour de la substitution subsistent entre le nouvel employeur et le personnel. Sauf cas de force majeure, la cessation de l'activité de l'entreprise ou de l'établissement ne dispense pas l'employeur de respecter les règles prévues en matière de résiliation des contrats. ${ }^{55}$

Il ressort de cet article que lorsqu'il y a substitution de l'employeur tous les contrats de travail existant en ce moment précis continuent à demeurer avec le nouvel employeur.

Et l'art.62 du code du travail énonce qu'un contrat de travail à durée indéterminée ne peut être résilié que pour un motif valable lié à l'aptitude ou à la conduite du travailleur sur les lieux de travail dans l'exercice de ses fonctions ou fondé sur les nécessités du fonctionnement de l'entreprise, de l'établissement ou du service. Il conviendrait, aussi, d'ajouter l'article 78 du code du travail qui donne la possibilité de résilier un contrat pour des raisons économiques.

D'où la décision de rompre certains contrats de travail en cours au moment de la transformation peut paraître comme du licenciement abusif. Le fait de choisir les travailleurs qui devraient partir sur aucun critère objectif et de leur payer un décompte final qui d'ailleurs

54 Au cours de notre entretien avec son conseiller juridique, il a eu beaucoup de peines pour nous expliquer les véritables raisons de cette sélection. Il invoque tantôt les rapports de chef de chaque département auxquels il suppose liés la performance du travailleur ou sa productivité tantôt les travailleurs en âge d'être mis en retraite.

55 Art. 80 du code de travail de 2002 . 
ne correspond pas au calcul que l'on doit faire sur le décompte, peut paraître comme une manœuvre fallacieuse tendant à nuire le travailleur.

L'art.63 du code de travail dispose « La résiliation sans motif valable du contrat à durée indéterminée donne droit, pour le travailleur, à une réintégration. A défaut de celle-ci, le travailleur a droit à des dommages- intérêts fixés par le Tribunal du travail calculés en tenant compte notamment de la nature des services engagés, de l'ancienneté du travailleur dans l'entreprise, de son âge et des droits acquis à quelque titre que ce soit. Toutefois le montant de ces dommages-intérêts ne peut être supérieur à 36 mois de sa dernière rémunération. ${ }^{56}$ En vertu de cet article les travailleurs de l'ancienne marsavco doivent normalement être réintégrés dans la nouvelle s'ils estiment être lésés. Au cas contraire la nouvelle marsavco doit leur payer les dommages et intérêts.

« Le travailleur a la faculté de réintégrer l'entreprise ou non car dans cette hypothèse, la réintégration est un droit et non une obligation. Il a été jugé que l'employeur ne peut se soustraire au paiement des dommages-intérêts pour la rupture abusive du contrat de travail en invoquant une décision de réintégration prise par lui mais rejetée par le travailleur qui la considère comme un piège. $\|^{57}$ « En ce qui concerne l'ancienneté en cas de substitution d'employeur, c'est l'unité économique qui compte et non l'unité juridique. Donc l'ancienneté continue et mettre fin au contrat pour supprimer l'ancienneté serait une violation de la loi. $»^{58}$

«Les contrats de travail ne peuvent déroger aux dispositions d'ordre public définies par la législation et la réglementation en vigueur. Toute clause contractuelle accordant au travailleur des avantages inférieurs à ceux prescrits par le présent Code est nulle de plein droit. $)^{59}$

Normalement la transaction est un mode de règlement de litige. Mais dans la pratique de plus en plus les parties l'utilisent pour mettre fin au contrat de travail à l'amiable. Dans ce cas les droits fondamentaux du salarié doivent être respectés. Et la jurisprudence soutient que la clause transactionnelle ne dispense pas l'employeur de ses obligations envers le travailleur. Celui-ci peut à tout moment saisir le Tribunal du Travail s'il estime être lésé dans ses droits. En d'autres mots la clause transactionnelle n'est pas un garde-fou auquel l'employeur peut se prémunir. Le travailleur de l'ancienne marsavco peut à tout moment saisir le Tribunal du Travail s'il estime être préjudicié dans ses droits. Le comportement de la nouvelle marsavco peut cacher la crainte du calcul des décomptes colossaux si l'on continuait à garder certains travailleurs. Leur ancienneté risque de peser lourd.

Cependant,si les raisons évoquées par la nouvelle marsavco sont valables, elle avait la possibilité de se fonder sur le code du travail à son article 62 ou à son article 78 qui lui

56 Art. 63 du code de travail de 2002.

57 Kumbu Ki Ngimbi, Droit social (droit du travail et de la sécurité sociale), manuel d'enseignement, Kinshasa, 2015, p.65.

58 Kumbu Ki Ngimbi, note 1, p.65.

59 Art. $37 \mathrm{du}$ code de travail de 2002. 
permet de procéder à un licenciement lié au motif économique en obtenant l'autorisation du Ministre de l'Emploi,du Travail et de la Prévoyance Sociale.

Il conviendrait de savoir que ce licenciement est tributaire de l'arrêté ministériel n 12 / CABMIN/TPS/116/2005 du 26 octobre 2005 tel que complété par l'arrêté n¹2/CAB-MIN/ ETPS/038/08 du 08/08/2008 portant interdiction provisoire de licenciement massif par l'Inspecteur du travail.

\section{Conclusion}

Les sociétés peuvent connaître des mutations mais cela ne veut pas signifier qu'à cette occasion les droits des travailleurs doivent être ignorés.

Le code travail est élaboré dans le sens de la protection surtout des travailleurs mais aussi de l'employeur. Il est l'instrument par lequel l'autorité publique assainit l'univers du travail et y instaure la paix.D'où il mérite d'être appliqué.

\section{Bibliographie}

\section{TEXTES OFFICIELS}

La constitution de la RDC, journal officiel de la RD Congo, 47ième année, numéro spécial du 18 février 2006.

La loi ${ }^{\circ} 015 / 2002$ du 16 octobre2002 portant code du travail,journal officiel de la RD Congo,numéro spécial du 15 juillet 2002.

Code civil Congolais livre 3

Acte uniforme relatif au droit des Sociétés Commerciales et du Groupement d'Intérêt Economique, journal officiel d'Ohada, n² du $1^{\text {er }}$ octobre 1997.

\section{DOCTRINES : ouvrages et autres documents.}

Alioune Dièye, Régime juridique des sociétés commerciales et du GIE dans l'espace ohada, 4 ième éd., 2014.

Belgeo (Revue belge de géographie), https://belgeo.revues.org/11572, consulté le 1 mars 2016.

Code de travail, http : // www.collectif24.org/lib/documents/code_travail-2.pdf, consulté le 10 octobre 2014.

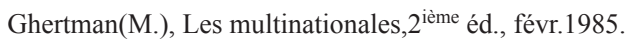

John Van Damme, Extraits des études sur les sociétés commerciales, in revue de doctrine et de jurisprudences coloniales, 1926.

Kumbu ki Ngimbi, Droit du travail, manuel d'enseignement, Galimage, Kinshasa, 2010.

Kumbu Ki Ngimbi,Droit social(droit du travail et de la sécurité sociale),manuel d'enseignement,Galimage, $3^{\text {ième éd., } 2015 .}$

Lelo-Di-Makangu (U.), La capitalisation du Groupement d'Intérêt Economique de l'Ohada, http:// ww w.mémoireonline.com, consulté le 04 mars 2016 
Lukombe Ghenda, Droit congolais des sociétés, t.2, Presses Universitaires au Congo, Kinshasa, 1999. Luwenyema Lule, «Précis de droit du travail congolais », Kinshasa, 1987, p.16

Masanga Phoba Mvioki (J.), notes polycopiées de droit du travail, UNIKIN, KINSHASA, 2010.

Moreau (A.), La société anonyme. Traité Pratique, Librairie du journal des notaires et avocats, $2^{\text {ième }}$ éd.,Paris.

Serges (J.), Les conditions de la croissance économique, Centre d'étude pour l'action social, KinshasaZaïre, Sept.1973.

Stéphanie Nsomwe, réflexion sur la protection de l'emploi dans le cadre de la sous-traitance au regard du code du travail en RDC. Cas de travailleurs assujettis par la sous-traitance dans le secteur de télécommunications, Librairies KAS, 2015.

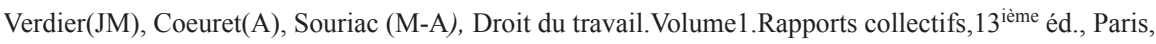
2005.

Wahl(M.), De la transformation des sociétés, J.SOC., 1910 


\section{Impressum}

\section{KAS African Law Study Library - Librairie Africaine d'Etudes Juridiques}

Herausgeber: Konrad-Adenauer-Stiftung e.V., Klingelhöferstraße 23, 10785 Berlin

Schriftleitung: RA Prof. Dr. Hartmut Hamann, Falkertstraße 82, D-70193 Stuttgart Telefon: +49 (o) 711 120950-30 | Fax : +49 (o) 711 120950-50

Mail: hamann@hamann-legal.de

Erscheinungsweise: 4 Ausgaben pro Jahr

Druck und Verlag: Nomos Verlagsgesellschaft mbH \& Co. KG - Waldseestr. 3-5 D-76530 Baden-Baden. Telefon +49-7221-2104-0 / Fax 49-7221-2104-27

E-Mail:nomos@nomos.de

Anzeigen: sales friendly Verlagsdienstleistungen - Inh. Frau Bettina Roos - Pfaffenweg 15-D-53227 Bonn Telefon +49-228-97898-o / Fax +49-228-97898-20 E-Mail: roos@sales-friendly.de

Urheber- und Verlagsrechte: Die Zeitschrift und alle in ihr enthaltenen einzelnen Beiträge sind urheberrechtlich geschützt. Jede Verwertung außerhalb der engen Grenzen des Urheberrechtsgesetzes ist ohne Zustimmung des Verlages unzulässig. Das gilt insbesondere für Vervielfältigungen, Übersetzungen, Mikroverfilmungen und die Einspeicherung und Verarbeitung in elektronischen Systemen. Der Nomos Verlag beachtet die Regeln des Börsenvereins des Deutschen Buchhandels e.V. zur Verwendung von Buchrezensionen.

ISSN $2363-6262$

\section{Nomos}

